\title{
A Novel Tumor-Suppressor, CDH18, Inhibits Glioma Cell Invasiveness Via UQCRC2 and Correlates with the Prognosis of Glioma Patients
}

\author{
Ya-hui Baia Yun-bo Zhan ${ }^{\mathrm{a}} \quad$ Bin Yu Wei-Wei Wang $^{\mathrm{b}}$ Li Wang ${ }^{\mathrm{b}}$ Jin-qiao Zhou \\ Ruo-kun Chen ${ }^{\mathrm{a}}$ Feng-jiang Zhang ${ }^{\mathrm{a}}$ Xin-wei Zhao ${ }^{\mathrm{a}}$ Wen-chao Duan ${ }^{\mathrm{a}}$ \\ Yan-min Wang ${ }^{a}$ Jun Liua Jian-ji Bao ${ }^{a}$ Zhen-Yu Zhanga Xian-zhi Liua \\ aDepartment of Neurosurgery, The First Affiliated Hospital of Zhengzhou University, Zhengzhou, \\ ${ }^{b}$ Department of Pathology, The First Affiliated Hospital of Zhengzhou University, Zhengzhou, China
}

\section{Key Words}

Glioma $\cdot \mathrm{CDH} 18 \cdot \mathrm{UQCRC2} \cdot$ Invasion • Prognosis

\begin{abstract}
Background/Aims: $\mathrm{CDH} 18$ (cadherin 18) is specifically expressed in the central nervous system and associated with various neuropsychiatric disorders. In this study, the role of $\mathrm{CDH} 18$ in glioma carcinogenesis and progression was investigated. Methods: The expression of $\mathrm{CDH} 18$ and its prognostic value in patients with gliomas were analyzed in public database and validated by real-time PCR/immunohistochemical staining (IHC) in our cohort. CCK-8 assay, transwell migration assay, wound healing assay, clonogenic assay and tumorigenicity assay were used to compare the proliferation, invasion and migration ability of glioma cells with different expressions of CDH18. iTRAQ-based quantitative proteomic analysis were used to reveal the downstream target of $\mathrm{CDH} 18$. Rescue experiments were conducted to further validate the relationship between UQCRC2 and CDH18. Results: The expression of CDH18 was depressed in a ladder-like pattern from normal tissues to WHO IV gliomas, and was an independent prognostic factor in TCGA (The Cancer Genome Atlas), CGGA (the Chinese glioma genome-atlas) and our glioma cohorts $(n=453)$. Functional experiments in vitro and in vivo demonstrated that $\mathrm{CDH} 18$ inhibited invasion/migration, enhanced chemoresistance and suppressed tumorigenicity of glioma cells. UQCRC2 was identified as the downstream target of $\mathrm{CDH} 18$ by proteomic analysis. The expression of UQCRC2 was gradually absent as the WHO grades of gliomas escalated and was positively correlated with the expression of CDH18. Furthermore, in vitro assays demonstrated that down-regulation of UQCRC2 partly reversed the inhibition of invasion/migration ability and chemoresistance in $\mathrm{CDH} 18$ overexpressed glioma cell lines. Survival analysis demonstrated that combined CDH18/UQCRC2 biomarkers
\end{abstract}

Y.-h. Bai and Y.-b. Zhan contributed equally to this work.

Xian-zhi Liu, M.D.

and Zhen-Yu Zhang, M.D.
Department of Neurosurgery, First Affiliated Hospital of Zhengzhou University

Jian she Dong Road 1, Zhengzhou, Henan, 450001 (China)

E-Mail xzliu06@126.com; neurozzy@foxmail.com 
significantly influenced the prognosis of glioma patients. Conclusions: The present research demonstrated that $\mathrm{CDH} 18$ exerted its tumor-suppressor role via UQCRC2 in glioma cells and $\mathrm{CDH} 18$ might serve as a therapeutic target for treating gliomas.

(C) 2018 The Author(s)

Published by S. Karger AG, Basel

\section{Introduction}

Gliomas are the most common primary central nervous system(CNS) tumors in adults [1]. According to the newest WHO classification of tumors of CNS, gliomas were categorized into four grades (WHO Grade I to IV) on the basis of histologic criteria [2]. Particularly, the glioblastoma multiforme (GBM, WHO Grade IV) is considered to be one of the most malignant tumors in human cancers [3]. One of the main obstacles of treating gliomas is that the tumor is highly invasive which leads to rapid recurrence or progression [4]. Therefore, it is necessary to discover new functional targets in glioma carcinogenesis and develop effective biological molecular therapies correspondingly.

Cadherins, which include type I and type II cadherins, are integral membrane proteins and composed of six subfamilies of calcium-dependent cell adhesion molecules [5]. Type II cadherins are named due to the lack of a HAV cell adhesion recognition sequence, which is specific to type I cadherins [5]. Cadherins were reported to be involved in neural tube regionalization, neuronal migration, gray matter differentiation as well as neural circuit formation, and their abnormal expression was observed in several human cancers [6-8]. The CDH18 gene encodes a type II classical cadherin (cadherin 18, CDH18) that mediates calcium-dependent cell to cell adhesion. The mature cadherin proteins are comprised of a highly conserved C-terminal cytoplasmic domain, a single membrane-spanning domain, and a large N-terminal extracellular domain [5]. CDH18 is specifically expressed in the central nervous system and participates in the biological process such as synaptic adhesion, axon outgrowth and guidance [9]. Several studies had demonstrated that a genetic abnormality of $C D H 18$ was associated with various neuropsychiatric disorders such as bipolar disorder, schizophrenia [10,11], depression [12] and autism [13]. Additionally, germline mutations of CDH18 was revealed in sporadic colorectal cancers $[14,15]$ and keratocystic odontogenic tumors [16]. However, to our knowledge, no further research on the function of CDH18 in carcinogenesis was reported. Moreover, although CDH18 is specifically expressed and functional in the central nervous system, the role of CDH18 in gliomas was not reported in the current literature.

Apart from the WHO grades, age at diagnosis has been established as a vital parameter for the prognosis of patients with anaplastic astrocytoma and glioblastoma [17]. Moreover, recent studies have demonstrated that several genetic changes such as IDH1 mutation, single nucleotide polymorphisms (SNPs) in TERT and RTEL1 were strongly associated with age at diagnosis, which may partly explain the prognostic significance of age in gliomas $[18,19]$. Hence, there may exist other genetic differences between older group and younger group of patients with glioma, contributing to worse prognosis in older patients. In the current research, we used bioinformatics to profile the gene expression signatures in different age groups of gliomas in TCGA database for the first time and revealed that the expression and copy number variations (CNVs) of $\mathrm{CDH} 18$ was significantly down-regulated in the older group of gliomas than that of the younger group. We first demonstrated that the expression of $C D H 18$ was depressed in a ladder-like pattern from normal tissues to WHO IV gliomas, and was an independent prognostic factor in TCGA, CGGA and our glioma cohorts. We further performed in vitro and in vivo functional experiments, which demonstrated CDH18 inhibited glioma cell migration/invasion ability and enhanced chemoresistance. By using iTRAQbased quantitative proteomic analysis, we first identified ubiquinol-cytochrome c reductase core protein 2(UQCRC2) was a downstream target of CDH18 and proved the role of CDH18 on glioma cells was significantly dependent on UQCRC2. Last but not least, we revealed that the expression of UQCRC2 was gradually absent as the WHO grades of gliomas escalated and was positively correlated with the expression of CDH18. Survival analysis demonstrated 
that combined CDH18/UQCRC2 biomarkers significantly influenced the prognosis of glioma patients. These results showed that $\mathrm{CDH} 18$ was a tumor suppressor in glioma carcinogenesis and suggested CDH18 might serve as a novel therapeutic target for gliomas.

\section{Materials and Methods}

\section{Informed consent}

Informed consent was obtained from all individual participants included in the study.

\section{Ethical approval}

All procedures performed in studies involving human participants were in accordance with the ethical standards of the institutional research committee of the First Affiliated Hospital of Zhengzhou University.

\section{Patients, tissue specimens and clinical data}

Information of 595 lower-grade gliomas(LGG) and $160 \mathrm{GBM}$ cases was collected from database of TCGA (https://cancergenome.nih.gov). Data of 181 LGG and 144 GBM cases was extracted from CGGA (http:// www.cgga.org.cn). These data included: (1) RNA sequencing, (2) DNA copy-number variations(CNVs) and (3) Clinical data. Fresh-frozen tissues including 14 cases of WHO Grade II gliomas, 15 cases of WHO Grade III gliomas, 19 cases of GBM and 9 cases of normal brain tissues (collected in brain trauma surgery) were gathered in The First Affiliated Hospital of Zhengzhou University between August 2016 and November 2016. All patients had signed informed consents before tissue collection. A total of 527 patients were followed up from 2011 to 2014 at the First Affiliated Hospital of Zhengzhou University, and survival data of 453 patients was successfully collected, with the missing rate being $14.1 \%$ and the median duration of follow-up being 25.9 months. Clinical data such as sex, age, extent of resection, postoperative primary radiation therapy, postoperative primary chemotherapy, and WHO grades were collected from medical records. The survival data was acquired by telephone-calls or out-patient clinic.

\section{RNA extraction, reverse transcription, and quantitative real-time PCR ( $q R T P C R$ )}

The total RNA of the tissue specimens (14 cases of WHO Grade II gliomas, 15 cases of WHO Grade III gliomas, 19 cases of GBM and 9 cases of normal brain tissue) was extracted with TRI-zol reagent (Invitrogen, Carlsbad, CA, USA) and reversely transcribed with oligo(dT) primers as well as Super Script II (Invitrogen). mRNA level was quantified by QRT PCR with Quant SYBR Green PCR (Tian-Gen, Beijing, China) and normalized Glyceraldehyde 3-phosphate dehydrogenase (GAPDH) with the following primers:

CDH18 (Forward): 5'-AATGACAATCCACCCGAAC-3',

CDH18 (Reverse): 5'-GGCTGTGTTATCTTCATTGTCC-3',

GAPDH (Forward): 5'-TGATGACATCAAGAAGGTGGTGAAG-3',

GAPDH (Reverse): 5'-TCCTTGGAGGCCATGTGGGCCAT-3'.

The fold change was calculated by $2-\Delta \Delta \mathrm{CT}$.

\section{IHC of CDH18 and UQCRC2}

For immunostaining, a mouse anti-human monoclonal antibody for CDH18 (1:100 dilution, Proteintech, Chicago, USA) and UQCRC2 (1:200 dilution, Proteintech, Chicago, USA) were used according the manufacturer's instructions. IHC staining was evaluated by two independent pathologists under light microscopy. A positive immunoreactivity for CDH18/UQCRC2 was scored in four grades $(0,1,2$ and 3) depending on the intensity of the staining. The percentage of immunostaining positive cells was also scored in four categories: 0 (0\%), 1 (1 to 33\%), 2 (34 to 66\%) and 3 (67 to 100\%) [20]. The higher score of the two was adopted when discrepancies occurred. The sum of the intensity and percentage scores was considered as the final staining score. X-tile software (see "statistical analysis") was used to determine the cut-off values of the immunostaining scores for CDH18/UQCRC2. 


\section{Cellular Physiology Cell Physiol Biochem 2018;48:1755-1770 \\ \begin{tabular}{l|l|l} 
and Biochemistry Published online: August 2, 2018 & $\begin{array}{l}\text { (c) } 2018 \text { The Author(s). Published by S. Karger AG, Basel } \\
\text { www.karger.com/cpb }\end{array}$
\end{tabular}}

Bai et al.: Role of $\mathrm{CDH} 18$ in Glioma

\section{Cell culture and transfection}

The human glioma cancer cell lines U87 and U251 were obtained from the Cell Bank of the Shanghai Branch of the Chinese Academy of Sciences (Shanghai, China) and maintained with 10\% fetal bovine serum (FBS) and antibiotics in a humidified atmosphere containing $5 \%$ carbon dioxide at $37{ }^{\circ} \mathrm{C}$. The transfection was performed using Lipofectamine 3000 reagent (Invitrogen, Carlsbad, CA, USA) according to the manufacturer's instructions. CDH18 cDNA was cloned into the CMV- -PGK-puromycin vector (Genomeditech, Shanghai, China). shRNA targeting UQCRC2 (shUQCRC2, the target sequence of shUQCRC2 was 5'-GGCTTGGTGATTGCTTCTTTG-3') was synthesized and cloned into pLVX-Neo-IRES-ZsGreen1 (Genomeditech). Puromycin was used to establish the CDH18 stably transfected cell lines. Fluorescence was detected for selection of clones with stable inhibition of UQCRC2 expression. Stably transfected cell lines were validated by immunoblotting and used for further in vitro and in vivo experiments.

\section{Western Blotting}

Total protein was extracted from glioma cell lines with the total protein extraction kit (Millipore, Billerica, MA, USA) according to the manufacturer's instructions. Fifty micrograms of sample were first electrophoresed on a $20 \%$ SDS-polyacrylamide gel and then transferred to polyvinylidene difluoride (PVDF) membrane. Then, the PVDF membranes were incubated in blocking solution consisting of 5\% powered milk in TBST $(100 \mathrm{mM}$ Tris-HCI, $1.5 \mathrm{M} \mathrm{NaCl}$, and $0.5 \%$ Tween $20,1: 10$ dilution) at room temperature for $1 \mathrm{~h}$ and immunoblotted with CDH18 (1:500 dilution, Proteintech, Chicago, USA) antibody, UQCRC2 (1:1000 dilution, Proteintech, Chicago, USA) antibody and $\beta$-Actin antibody (1:1000 dilution, Abcam) overnight at 4 ${ }^{\circ} \mathrm{C}$, respectively. After incubation with 3, 3'-diaminobenzidine (DAB) solution, a microscope with a brightfield illumination was used to visualize the staining of tissues.

\section{CCK-8 assay}

Cell proliferation was measured with an CCK-8 assay according to the manufacturer's instructions as described before [21]. Stably transfected U87 and U251 cells were seeded at a density of $10^{3} /$ well into 96well culture plates for 24, 48, 72, 96 and $120 \mathrm{~h}$. For the CCK-8 assay, the cells were further incubated with 10ul CCK-8 (Sigma, Santa Clara, USA) for 4h. The absorbance was measured at $450 \mathrm{~nm}$.

\section{Transwell migration assay}

PBS (1:6) was used to dilute Matrigel (BD, New Jersey, USA) by placing the solution at $4^{\circ} \mathrm{C}$ overnight. The upper and lower chambers of each transwell chamber were added serum-free culture base. The processed cells $\left(100 \mathrm{ul}, 1 \times 10^{6} / \mathrm{ml}\right)$ were added into the transwell chambers and the lower chambers contained a serumfree culture base (500ul). $48 \mathrm{~h}$ later, the transwell chamber was taken out. Then the medium was extracted and the Matrigel gel and cells in upper chambers were wiped away with cotton swab. Then the lower chamber was added into $10 \%$ cold methanol and fixed for $30 \mathrm{~min}$ at room temperature. Then stationary liquid was extracted. The chamber was cleaned with PBS and stained with DAPI. Finally, the membrane at the bottom of the transwell chamber was cut down to count the cell number in the lower room.

\section{Wound healing assay}

The processed cells with $1 \times 10^{5} / \mathrm{ml}$ was inoculated into six plates. When the cell grew to confluence, the $100 \mathrm{ul}$ yellow pipette was used to scratch the bottom of the plate. Then the floating cells were washed away 3 times with PBS. The serum-free cell culture medium was added into plates and cultured for $24 \mathrm{~h}$. Samples and pictures of the plates were taken out and the results were analyzed. Relative mobility $(\%)=$ (distance between cells on both sides (0h)- distance between cells on both sides $(24 \mathrm{~h})$ )/ distance between cells on both sides $(0 \mathrm{~h})$.

\section{Clonogenic assay with temozolomide(TMZ) presence}

TMZ was purchased from Sigma Chemical (Sigma Chemical Co. St. Louis, Missouri, USA) and diluted to the desired concentration in DMEM at the time of use. Glioma cells in the logarithmic growth phase were digested into a single-cell suspension using a trypsin-EDTA solution, and $2 \mathrm{~mL}$ of a cell suspension was then seeded onto a 6 well cell culture plate at a density of 1000 cells $/ \mathrm{mL}$. The cells were cultured at $37^{\circ} \mathrm{C}$ in a well-humidified $95 \%$ air $/ 5 \%$ CO2 incubator for 14 days. On the $12^{\text {th }}$ day of cell culture, TMZ were added to the medium to a concentration at $20 \mathrm{ug} / \mathrm{ml}$. After $48 \mathrm{~h}$ of culture with the presence of TMZ, the colonies were 


\section{Cellular Physiology Cell Physiol Biochem 2018;48:1755-1770 and Biochemistry Published online: August 2, $2018 \quad \begin{aligned} & \text { DOI: 10.1159/000492317 } 2018 \text { The Author(s). Published by S. Karger AG, Basel } \\ & \text { www.karger.com/cpb }\end{aligned}$}

Bai et al.: Role of $\mathrm{CDH} 18$ in Glioma

fixed with $4 \%$ poly formaldehyde ( $5 \mathrm{ml}$ ) for 15 minutes and stained with GIMSA for 20 minutes. The colony formation was photographed and counted.

\section{Tumorigenicity assay in nude mice}

The processed cells with $1 \times 10^{6} / \mathrm{ml}$ were injected into the flank skin of nude mice. Then the animals were kept in constant temperature and sterile conditions. The health condition and tumor growth status of nude mice was observed every day. The longest diameter (a) and shortest diameter (b) of the subcutaneous tumor of nude mice was measured every 3 days. The formula: Tumor Volume $=a \times b^{2} / 2$. Finally, tumor volume was calculated, and the tumor growth curve was drawn. At the end of the 24 th day, all animals were sacrificed by cervical dislocation to acquire the tumor tissues.

\section{ITRAQ sample preparation}

Each sample was ultrasonically lysed with lysis buffer (containing $8 \mathrm{M}$ urea, 1\% Triton X-100, 65 Mm dithiothreitol, $1 \%$ protease inhibitor, $3 \mu \mathrm{M}$ Trichostatin A, 50mM Nicotinamide and 2mM EDTA). Cell debris was removed by centrifugation at $12000 \times \mathrm{g}$ at $4^{\circ} \mathrm{C}$ for $10 \mathrm{~min}$. The supernatant was mixed with $15 \%$ trichloroacetic acid and kept for 2 hours at $4^{\circ} \mathrm{C}$. Supernatant was removed by centrifugation at $12000 \times \mathrm{g}$ at $4^{\circ} \mathrm{C}$ for $3 \mathrm{~min}$. The precipitate was cleansed with acetone 3 times and dissolved with urea. The protein concentration of each sample was measured using 2-D Quant kit (GE Healthcare, Boston, USA). Dithiothreitol was added to the aliquot of protein with its concentration at $10 \mathrm{mM}$ for $1 \mathrm{~h}$. Indole-3-acetic acid(IAA) was then added with its concentration at $20 \mathrm{mM}$, and the samples were incubated in the dark for $30 \mathrm{~min}$. The concentration of urea in the samples was diluted to $2 \mathrm{M}$, with the triethylammonium bicarbonate(TEAB) concentration at $100 \mathrm{mM}$. Fifty micrograms of protein from each sample was digested with $0.2 \mathrm{~mL}$ of a $50 \mu \mathrm{g} /$ $\mathrm{mL}$ trypsin solution (Promega, Madison, WI, USA) at $37{ }^{\circ} \mathrm{C}$ overnight and $0.1 \mathrm{~mL}$ of a $50 \mu \mathrm{g} / \mathrm{mL}$ trypsin solution at $37{ }^{\circ} \mathrm{C}$ for $4 \mathrm{~h}$. The pooled samples (U87-NC, U87-CDH18, U251-NC, U251-CDH18) were labeled with $113,114,115$, and 116 iTRAQ reagents respectively, according to the manufacturer's protocol (AB SCIEX, Framingham, Massachusetts, USA). The samples then were mixed equally and dried by vacuum centrifugation.

\section{Two dimensional LC-MS/MS}

The labeled samples were firstseparated using a high performance reverse phase liquid chromatography column (Agilent 300 Extend C18 column, $5 \mu \mathrm{m}$ particles, $4.6 \mathrm{~mm}$ ID, $250 \mathrm{~mm}$ length, Agilent, California, USA). The samples were loaded onto the column in the buffer $(2 \%-60 \%$ acetonitrile, $10 \mathrm{mM}$ ammonium formats, $\mathrm{pH}=10$ ) for $80 \mathrm{~min}$. The eluted peptide was collected at one fraction per minute, and the 80 total fractions were pooled into 20 samples. The collected fractions were then dried by vacuum centrifugation. A total of 20 fractions were analyzed by LC-MS/MS. LC-MS/MS analysis was performed on a Q Exactive ${ }^{\mathrm{TM}}$ Plus mass spectrometer (Thermo Fisher Scientific, San Jose, CA). Peptides were dissolved in 0.1\% formic acid water and were loaded onto an Acclaim Pep Map RSLC reversed phase(RP) analytical column (Thermo Fisher Scientific, San Jose, CA) for separation. The flow rate used for RP column separation was 300nl/min with buffer A ( $0.1 \%$ formic acid water, $2 \%$ acetonitrile). Elution was performed with Buffer B $(0.1 \%$ formic acid water, $98 \%$ acetonitrile) as a gradient. The proportion of Buffer B increased from $7 \%$ to $20 \%$ in 24 $\mathrm{min}$, to $35 \%$ in $8 \mathrm{~min}$, to $80 \%$ in $5 \mathrm{~min}$ and a final hold in $80 \%$ buffer B for $3 \mathrm{~min}$. The mass spectrometer was operated in positive ion mode, and the mass spectrometry data was obtained using a data-dependent acquisition mode that selected the most abundant precursor ions from the survey scan $(350-1800 \mathrm{~m} / \mathrm{z})$ for high-energy collisional dissociation (HCD) fragmentation. The threshold for HCD fragmentation was set to $3 \mathrm{E} 3$ and the automatic gain control target was set to $3 \mathrm{E} 4$. Survey scans were acquired at a resolution of 70000 at $200 \mathrm{~m} / \mathrm{z}$, the HCD spectra resolution was set to 17500 at $200 \mathrm{~m} / \mathrm{z}$, and the normalized collision energy was $30 \mathrm{eV}$.

\section{Proteomics data analysis}

Mascot software (version 2.5, Matrix Science, London, UK) was used for database searching of all samples. The database was set to Swissport human database and the digestion enzyme was set to trypsin. The parent mass tolerance was $10 \mathrm{ppm}$ and fragment ion were 0.02Da. Scaffold (version Scaffold 4.0.7, Proteome Software Inc., Portland, OR, USA) was used for protein identification. The false discovery rate $($ FDR $)<1 \%$ was set on both peptide and protein level. The differentially expressed proteins (DEPs) were 
selected in the ITRAQ experiment based on the following criteria: unique peptides $\geq 1, \mathrm{P}$-value $<0.05$ and fold change $>1.5$ (or<0.67). The DEPs were annotated using the Gene Ontology (G0) database (http://www. geneontology. org/). Kyoto Encyclopedia of Genes and Genomes (KEGG) pathway analysis of the DEPs was conducted using the KEGG database (http://www.genome.jp/kegg/).

\section{Statistical analysis}

The Student's t-test was adopted to test for differences in the comparison of each two-groups. Oneway ANOVA was used to determine differences among at least three groups, with Bonferroni correction as the post hoc test. Overall survival (OS) was measured from the date of diagnosis to the date of death or last follow-up. Differences in survival between two groups of patients were assessed using the Kaplan-Meier method and analyzed using the log-rank test in the univariate analysis. X-tile 3.6.1 (http://medicine.yale. edu/lab/rimm/research/software.aspx) was used to determine the cut-off values in univariate analysis. Suitable prognostic factors were put into Cox proportional hazards regression model to identify independent prognostic factors (multivariate analysis). To determine functional gene sets, gene set enrichment analyses were performed using a comprehensive set of functional annotation tools (The Database for Annotation, Visualization and Integrated Discovery, DAVID). All data are presented as the Mean \pm SD. P values less than 0.05 are considered statistically significant. Data statistics were performed using software Graph-pad prism 5 (Graph-pad Inc, La Jolla, USA) and IBM SPSS Statistics 19(IBM Corp., Armonk, NY, USA).

\section{Results}

\section{Identification of CDH18 in the TCGA glioma cohort}

Older age at diagnosis was established as a well-known predictor of worse prognosis in GBM. In addition, genetic differences exist between the older group and younger group of glioma patients. Therefore, we compared the expression profile of genes between the older group and a younger group of GBM patients in TCGA database. RNA sequences and clinical data of TCGA GBM cohort $(\mathrm{n}=160)$ were acquired by downloading Bioconductor/TCGA biolinks function package from TCGA (https://tcga-data.nci.nih.gov/tcga/). The cohort was divided into older group (age $\geq 65$ years, $n=59$ ) and younger group (age $<65$ years, $n=101$ ). After pretreatment, expression data of 17452 genes were acquired. We used T-test and DESeq package [22] to analyze the differential expressed genes (DEGs). Significant P-value was set as 0.05 . The absolute value of $\log _{2}$ FoldChange was set as 1 . There were 71 DEGs generated by T-test and 46 DEGs generated by DESeq, with an intersection of 33 DEGs and a union of 84 DEGs, which was depicted in a Venn diagram (Fig. 1A). The 33 genes were further displayed in a hierarchical clustering heatmap (Fig. 1B). Gene Ontology (GO) analysis of the 84 genes revealed that these DEGs were mainly enriched in the biological process (BP) of cell adhesion, in the cellular content (CC) of integral to membrane, and in the molecular function (MF) of calcium ion binding (Fig. 1C). Copy number variations (CNVs) have considerable impact on the viability of oncogenes or tumor-suppressor genes. We used Genomic Identification of Significant Targets in cancer (GISTIC) [23] to analyze the CNVs between older and younger group of GBM. The amplifications/deletion threshold was set as 0.1 , and the qv threshold was set as 0.25 . The results demonstrated that there was considerable amplification in chromosome 7 and significant deletion in Chromosome 10 (Supplementary Fig. 1). For all supplemental material see www.karger.com/doi/10.1159/000492317. In order to determine the relationship between the CNVs and DEGs ( $n=33$, Fig. 1B), we used fisher's exact test to whether the 33 DEGs were correlated with the CNVs. The results showed that only the expression of $A D H 1 B, S L C 18 A 1$ and $C D H 18$ were significantly correlated with the CNVs (Supplementary Table 1). Moreover, we found that the mRNA expression level of CDH18 was significantly lower in older group $(n=92)$ than younger group $(n=572)$ of TCGA cohort $(\mathrm{P}<0.0001)$. This phenomenon was also observed between older group $(\mathrm{n}=11)$ and younger group $(\mathrm{n}=314)$ of CGGA cohort $(\mathrm{P}=0.0304)$. Combined the above results, CDH18 was chosen for further investigation in our study. 


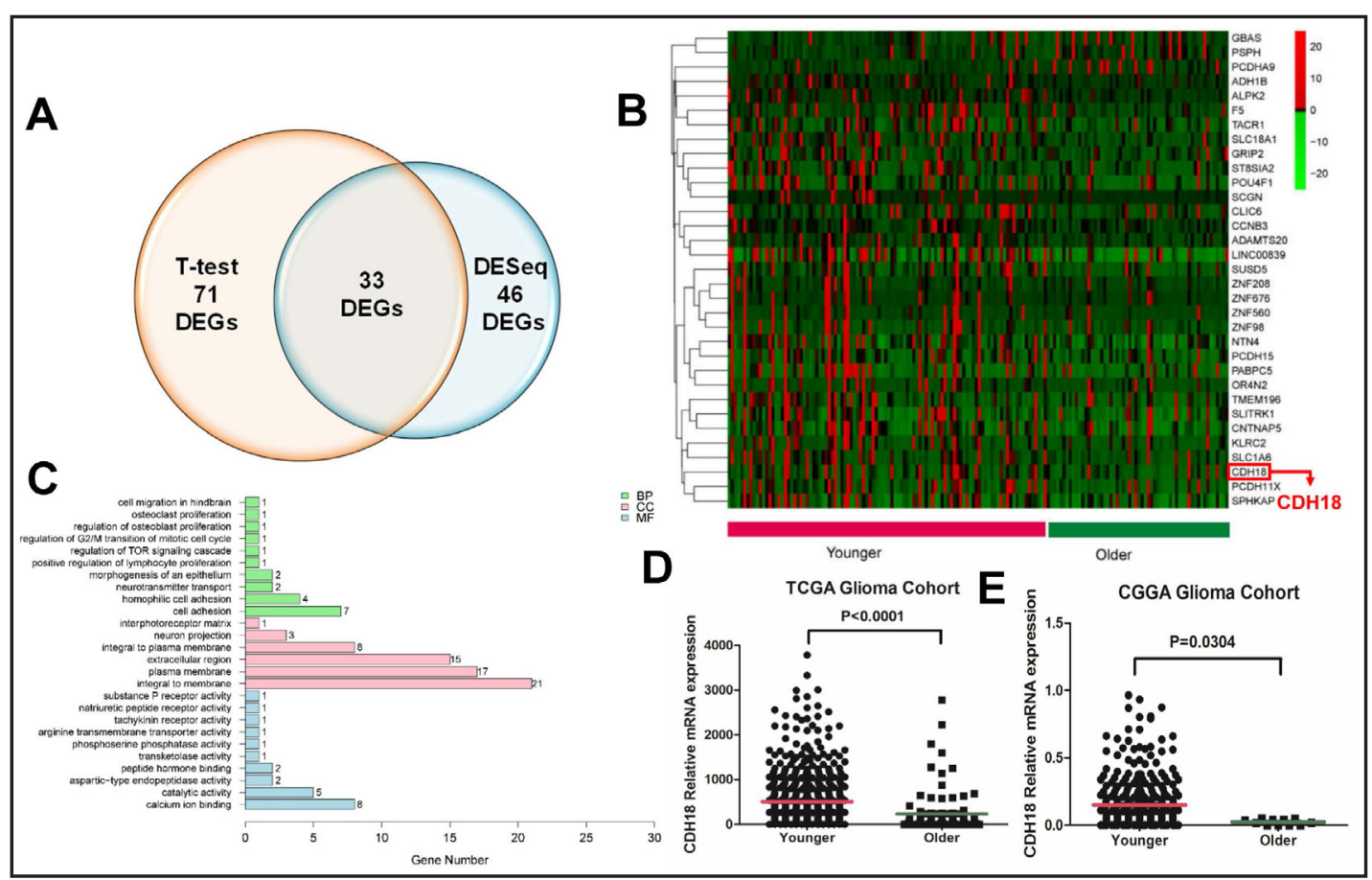

Fig. 1. Identification of CDH18 in the TCGA and CGGA glioma cohort. (A) Venn diagram showed 71 differentials expressed genes(DEGs) generated by T-test and 46 DEGs generated by DESeq, with an intersection of 33 DEGs and a union of 84 DEGs. (B) Heatmap showed the 33 DEGs between older and younger groups of GBM. Green represented lower mRNA expression levels and red represents higher expression levels than those in the control. The color scale shown at the upper right indicated the fold changes. (C) Gene Ontology(GO) analysis on the enrichment of 84 DEGs in biological process(BP), cellular content(CC) and molecular function(MF). (D) The mRNA level of CDH18 was compared between younger and older group of TCGA glioma cohort and (E) CGGA glioma cohort.

Table 1. Multivariate analysis of prognostic factors for OS in glioma patients $(n=453)$. OR: odds ratioCI:

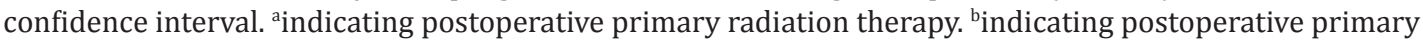
chemotherapy.

\begin{tabular}{lcc}
\hline Factors & OS & p-value \\
& OR(95\%CI) & $<0.001$ \\
Resection extent (Yes or No) & $1.690(1.264-2.259)$ & 0.014 \\
RT a (Yes or No) & $1.533(1.090-2.155)$ & 0.264 \\
CHT b (Yes or No) & $1.205(0.868-1.672)$ & $<0.001$ \\
Grade (II, III or IV) & $3.325(2.500-4.423)$ & 0.002 \\
Age ( $\leq 50>50$ ) & $1.541(1.167-2.034)$ & 0.006 \\
CDH18 (High or Low) & $0.608(0.426-0.867)$ & 0.108 \\
UQCRC2 (High or Low) & $1.362(0.935-1.986)$ & \\
\hline
\end{tabular}

\section{CDH18 is down-regulated in gliomas and correlated with favorable prognosis}

FireBrowse database (http://firebrowse.org/viewGene.html) showed that the expression of CDH18 was significantly lower in LGG and GBM, compared to normal brain tissue (Fig. 2A). Genotype-Tissue Expression database (GTEx, https://www.gtexportal.org/ home) revealed that the expression of CDH18 was much higher in brain tissue than those of other human tissue (Fig. 2B). TCGA database demonstrated that the expression of CDH18 was significantly lower in Grade IV gliomas than Grade III gliomas (Fig. 2C, $p<0.001$ ), and considerably lower in Grade II gliomas than normal brain tissue (Fig. 2C, $p<0.001$ ). Similarly, CGGA database showed the mRNA expression level of CDH18 was significantly lower in Grade 


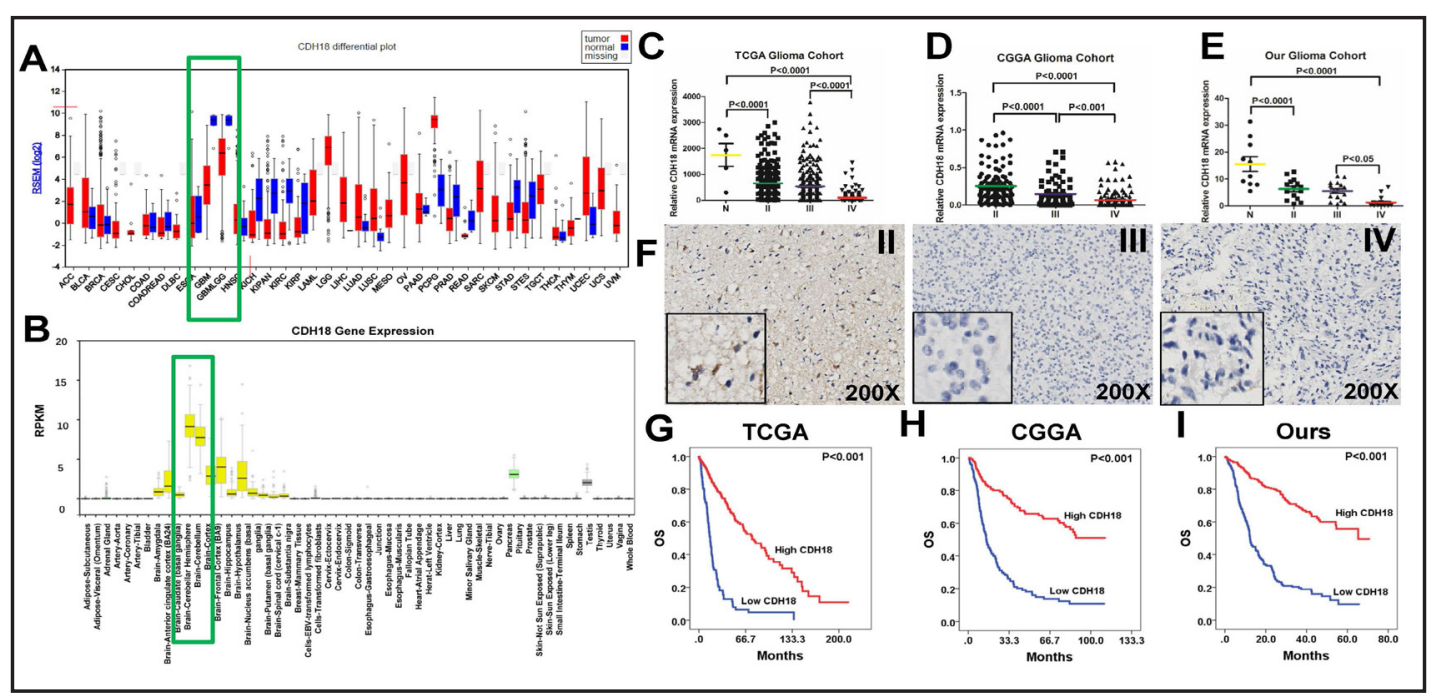

Fig. 2. CDH18 is down-regulated in gliomas and correlated with favorable prognosis. (A) CDH18 expression shown in the FireBrowse database. (B) CDH18 expression shown in the GTEx Portal. (C, D, E) Analysis on mRNA level expression of CDH18 in normal brain tissue, Grade II, Grade III and Grade IV gliomas from TCGA, CGGA and our cohorts by one-way ANOVA. (F) Immunohistochemical staining of CDH18 in Grade II, Grade III and Grade IV gliomas. (G, H, I) Univariate survival analysis of patients in TCGA, CGGA and our glioma cohorts according to CDH18 expression.

IV gliomas than Grade III gliomas (Fig. 2D, p<0.0001), and considerably lower in Grade III gliomas than Grade II gliomas (Fig. 2D, $\mathrm{p}<0.001)$. Results from our glioma cohort $(\mathrm{n}=453)$ showed that the mRNA expression of CDH18 was much lower in Grade IV gliomas than Grade III gliomas (Fig. 2E, $\mathrm{P}<0.05$ ), and significantly lower in Grade II gliomas than normal brain tissue (Fig. 2E, P<0.0001). Survival analysis of TCGA glioma cohorts and CGGA glioma cohorts showed that patients with high CDH18 mRNA expression had an obviously longer OS than that with low CDH18 expression (Fig. 2G, 2H, P<0.001). IHC of CDH18 in glioma FFPE tissues revealed that staining was localized mainly in the cytoplasm and cytomembrane, with the expression of $\mathrm{CDH} 18$ decreased as the WHO grades increased. Survival analysis of our glioma cohort revealed that patients with high CDH18 expression had an obviously longer OS than those with low CDH18 expression (Fig. $2 \mathrm{I}, \mathrm{P}<0.001$ ). Univariate analysis showed that age $(\mathrm{P}<0.001)$, extent of resection $(\mathrm{P}=0.007)$, RT $(\mathrm{P}<0.001)$, CHT $(\mathrm{P}<0.001)$, Grade $(\mathrm{P}<0.001)$, CDH18 $(\mathrm{P}<0.001)$ and UQCRC2 $(\mathrm{P}<0.001)$ were factors that impact the prognosis in patients with gliomas (Supplementary Table 2). Then these prognostic factors with significance were included in multivariate survival analysis. Results revealed that expression of CDH18 $(\mathrm{P}=0.006)$, resection extent $(\mathrm{P}<0.001)$, $\mathrm{RT}(\mathrm{P}=0.014)$, grade $(\mathrm{P}<0.001)$ and age $(\mathrm{P}=0.002)$ were significantly independent prognostic factors in patients with gliomas (Table 1 ).

\section{CDH18 inhibits glioma cell invasion/migration ability and enhances chemoresistance}

To better explore how CDH18 influences the biology of glioma cells, overexpression of $\mathrm{CDH} 18$ was forced by viral transfection. Western blotting showed that forced overexpression of CDH18 obviously increased the protein levels of CDH18, compare to those of control cell lines. Transwell assay was used to investigate the effect of CDH18 on the invasiveness of glioma cells. Results showed that the mean cell number of the U87-CDH18 group moved to the lower chamber was $(138.0 \pm 4.04)$, and the mean cell number of the U87-NC group moved to the lower chamber was $(255.7 \pm 4.81)$. There was significant statistical difference between the two groups (Fig 3B, P<0.0001). Similarly, in U251-CDH18 group, the mean number of cells moved to the lower chamber $(32.3 \pm 1.45)$ was significantly lower than that $(90.0 \pm 1.53)$ of U251-NC (Fig 3B, P<0.0001). Results of a wound healing assay showed that the scratch spacing between NC and CDH18 was shrinking along with time in U87 and U251 cell

\section{KARGER}


lines. Compared to the control group, the scratch spacing of experimental group with U87-CDH18 was much wider (NC vs $\mathrm{CDH} 18,0.28 \pm 0.031$ vs $0.13 \pm 0.12, \mathrm{P}=0.0111$, Fig $3 \mathrm{C}$ ). Similarly, the scratch spacing of experimental group with U251CDH18 was much wider (NC vs $\mathrm{CDH} 18,0.33 \pm 0.35$ vs $0.18 \pm 0.02$, $\mathrm{P}=0.0206$, Fig 3C). Therefore, the relative mobility from $0 \mathrm{~h}$ to $24 \mathrm{~h}$ in the CDH18 group was significantly decreased. Colony formation assay under TMZ presence $(20 \mathrm{ug} / \mathrm{ml})$ showed that the mean number of cell colonies of U87 and U251 cell lines with CDH18 were obviously decreased (U87-NC vs U87-CDH18: $1246.0 \pm 48.14$ vs $437.7 \pm 21.07, \quad \mathrm{P}<0.0001$, U251-NC vs U251-CDH18: $1130.0 \pm 34.07$ vs $449.0 \pm 26.56$, $\mathrm{P}<0.0001$, Fig 3D). CCK-8 assay showed that up-regulation of CDH18 did not significantly change the viability of U87 and U251 cell lines (OD values at $450 \mathrm{~nm}, \mathrm{U} 87-\mathrm{NC}$ vs U87-CDH18: $1.85 \pm 0.057$ vs $1.95 \pm 0.026$, $\mathrm{P}=0.2004$, U251-NC vs U251-

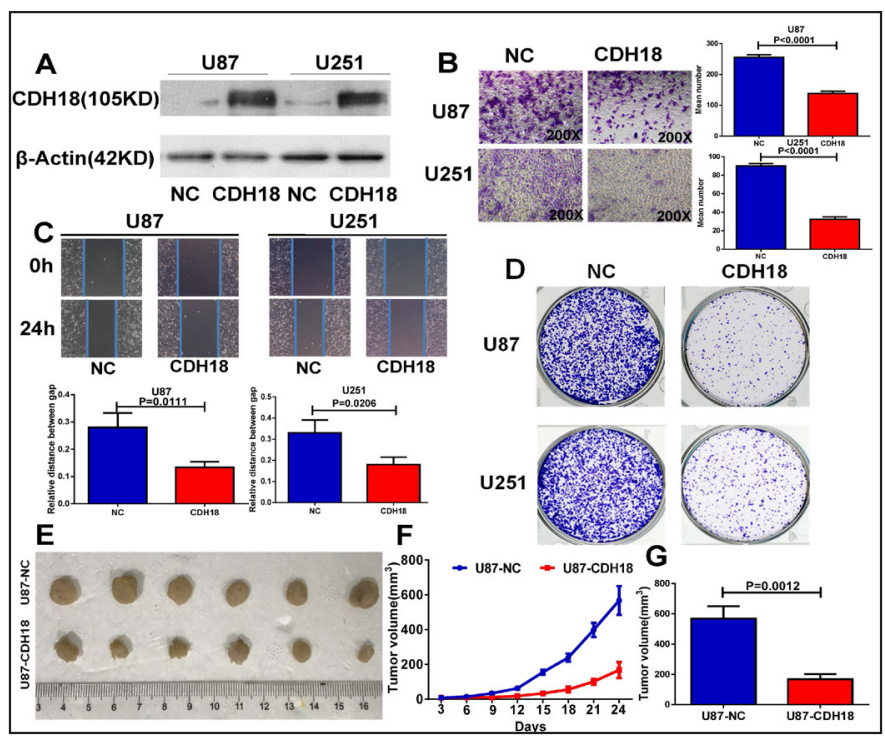

Fig. 3. CDH18 inhibited glioma cell invasion/migration, enhances chemoresistance, and suppressed tumorigenicity of glioma cells in vivo. (A) Relative expression of CDH18 in U87 and U251 cell lines by Western Blot. (B) The impact of stably up-regulating CDH18 expression on cell invasion in U87 and U251 glioma cell lines by transwell assay. (C) The impact of stably up-regulating CDH18 expression on cell invasion in U87 and U251 glioma cell lines by wound healing assay. (D) The impact of stably up-regulating CDH18 expression on chemoresistance in U87 and U251 glioma cell lines by colony formation assay of chemoresistance. (E, F, G) In vivo, tumorigenesis of U87-CDH18 group in nude mice. (E) Tumor imaging. (F) tumor growth curve of subcutaneous tumors in nude mice. (G) The impact of up-regulation CDH18 expression on tumorigenesis in U87 glioma cell lines.

CDH18: $1.81 \pm 0.048$ vs $1.76 \pm$

0.022, $\mathrm{P}=0.3764$, Supplementary Fig. 2A, 2B)

\section{CDH18 suppresses the tumorigenicity of glioma cells in vivo}

In order to test whether CDH18 inhibits glioma cell tumorigenicity in vivo, we designed a subcutaneous tumor test with nude mice. And we analyzed growth conditions of tumors and measured tumor volume. Results show that tumors with overexpression of CDH18 had a slower rate of growth and smaller volume than tumors with NC (Fig. 3E). Tumor tissue with U87-CDH18 could be observed in the eighth day, while tumor tissue with U87-NC could be observed in the fifth day. On the twenty-fourth day, volume of tumor with CDH18 was $167.82 \pm 34.96 \mathrm{~mm}^{3}$, while volume of tumor with $\mathrm{NC}$ was $568.4 \pm 82.30 \mathrm{~mm}^{3}$ (Fig. 3F, 3G). There was a statistically significant difference between the two groups (CDH18 vs NC, $\mathrm{P}=0.0012$ ).

\section{Identification of DEPs between CDH18 overexpression and control groups}

To identify the DEPs between CDH18 overexpression and control groups of glioma cell lines, total proteins from the four groups (U87-NC, U87-CDH18, U251-NC, U251-CDH18) were extracted for iTRAQ analysis. In total, 4975 proteins were detected using iTRAQ. Compared with the U87-NC group, the expression levels of 201 proteins were significantly different (upregulated $\geq 1.5$-fold or downregulated $\leq 0.67$-fold; $P \leq 0.05$ ) in the U87-CDH18 group. Compared with the U251-NC group, the expression levels of 331 proteins were significantly different (upregulated $\geq 1.5$-fold or downregulated $\leq 0.67$-fold; $\mathrm{P} \leq 0.05$ ) in the U251-CDH18 group. An intersection of 143 DEPs was identified between U87 (201 DEPs) 
and U251(331 DEPs) cell lines (Supplementary Fig. 3). A hierarchical clustering heatmap of the 143 DEPs is shown in Fig.4A.

\section{GO and KEGG pathway analyses of DEPS}

The identified DEPs were classified according to their biological processes (BP), cellular components (CC) and molecular functions (MF) using the David database (Fig.4B). In the BP category, lipid localization, intracellular transport and protein localization/targeting to mitochondrion were overrepresented. In the CC category, mitochondrial transport/ envelope/part/membrane were highlighted. In the MF category, hydrogen ion/monovalent inorganic cation transmembrane transporter activity and cytochrome-c oxidase activity were overrepresented. In KEGG pathway analysis, the top 5 pathways were Parkinson's disease, oxidative phosphorylation(OXPHOS), Huntington's disease, cardiac muscle contraction, and Alzheimer's disease pathways, in which UQCRC2 was the prior participant without exception (Supplementary Table 3, Fig.4C). Consequently, combined the results of proteomics, GO and KEGG pathway analyses, we select UQCRC2 as the downstream target of CDH18 for further validation with in vitro assays.

\section{Down-regulation of UQCRC2 partly reversed the tumor-suppressor role of CDH18 in glioma} cells

To explore how CDH18 and UQCRC2 influenced each other in gliomas cells, the expression of UQCRC2 was down-regulated by transfection. Western blotting showed that down-regulated of UQCRC2 decreased the protein levels of UQCRC2. Then we carried out the

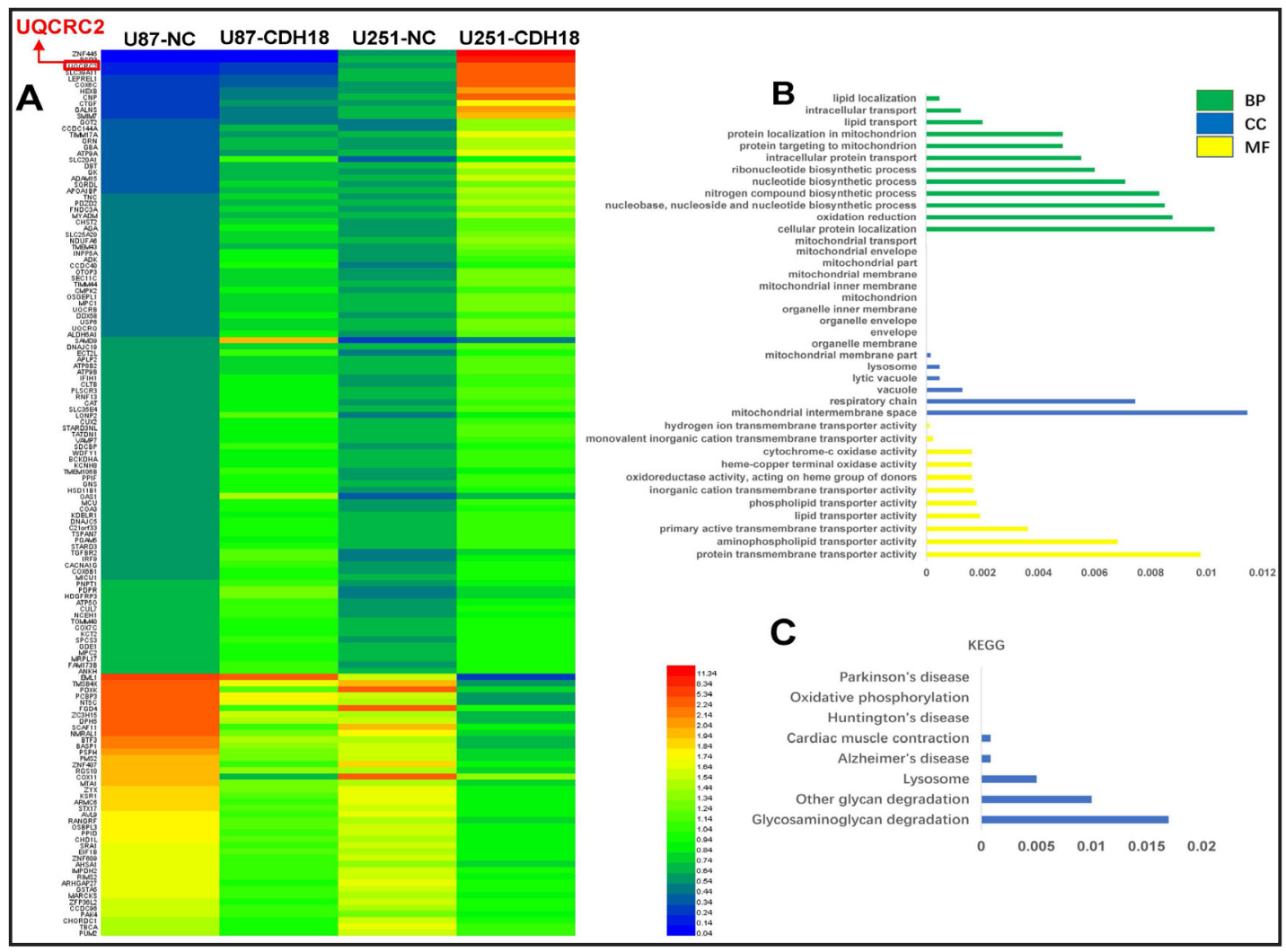

Fig. 4. Identification of differentially expressed proteins (DEPs) between CDH18 overexpression and control groups using iTRAQ-based proteomic analysis. (A) Heatmap showed the 143 DEPs between CDH18 overexpression (U87-CDH18 and U251-CDH18) and control (U87-NC and U251-NC) groups of glioma cell lines. Blue represented lower protein expression levels and red represents higher expression levels than those in the control. The color scale shown at the lower right indicates the fold changes. (B)GO analysis of the DEPs. (C) KEGG pathway analysis of the DEPs. 
transwell assay with CDH18 and shUQCRC2. Results show that the mean cell number of the U87-shUQCRC2 group that moved to the lower chamber was significantly increased compared to U87-CDH18 (CDH18 vs shUQCRC2, $124.3 \pm 7.22$ vs $219.3 \pm 4.91$, P=0.0004, Fig.5D). Similarly, in the U251-shUQCRC2 group, the mean number of cells that moved to the lower chamber was significantly increased than U251-CDH18 (CDH18 vs shUQCRC2, $40.3 \pm 4.91$ vs $62.3 \pm 4.05$, $\mathrm{P}=0.0259$, Fig.5D). Wound healing assay revealed that the relative mobility from $0 \mathrm{~h}$ to $24 \mathrm{~h}$ in shUQCRC2 group increased in the U87 and U251 cells lines (U87, CDH18 vs shUQCRC2, $0.14 \pm 0.007$ vs $0.24 \pm 0.003$, $\mathrm{P}=0.0002$, U251, CDH18 vs shUQCRC2, $\quad 0.16 \pm 0.021$ vs $0.29 \pm 0.02$, P=0.0108, Fig.5C). The wound healing assay showed that down-regulation of UQCRC2 partly reversed the tumor migration suppressor role of CDH18 in U87 and U251 glioma cell lines. In the transwell assay, downregulation of UQCRC2 partly reversed the tumor invasion suppressor role of $\mathrm{CDH} 18$ in U87 and U251 glioma cell lines. In the colony formation assay in the presence of TMZ, the number of cell colonies with shUQCRC2 increased (U87CDH18+shUQCRC2 vs U87-

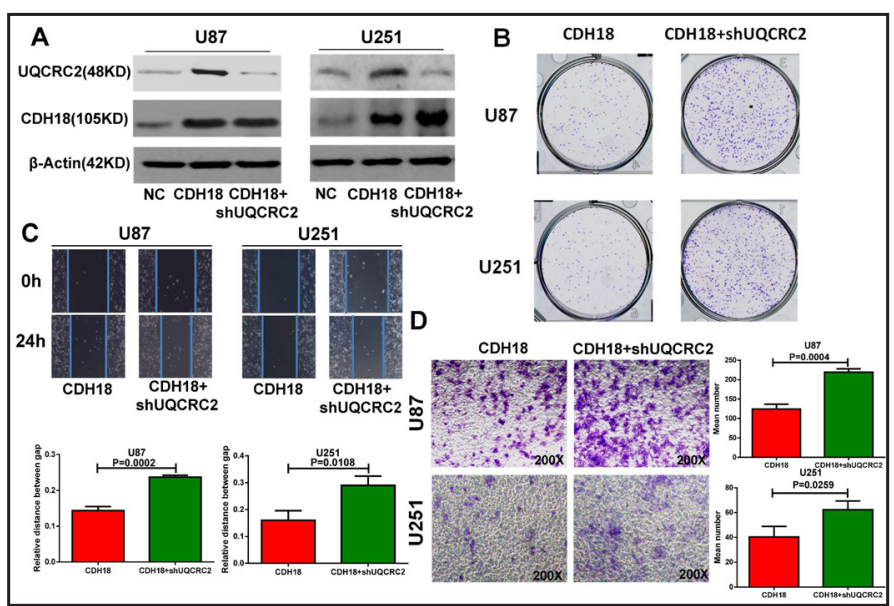

Fig. 5. Down-regulation of UQCRC2 partly reversed the tumor suppressor role of CDH18 in glioma cells. (A) Relative expression of CDH18 and UQCRC2 in U87 and U251 cell lines by Western Blot. (B) The impact of down-regulation of UQCRC2 on cell proliferation in U87 and U251 glioma cell lines with TMZ (20um/L) by colony formation assay of chemoresistance. (C) The impact of downregulation of UQCRC2 on cell migration in U87 and U251 glioma cell lines using a wound healing assay. (D) The impact of downregulation of UQCRC2 on cell invasion in U87 and U251 glioma cell lines using a transwell assay.

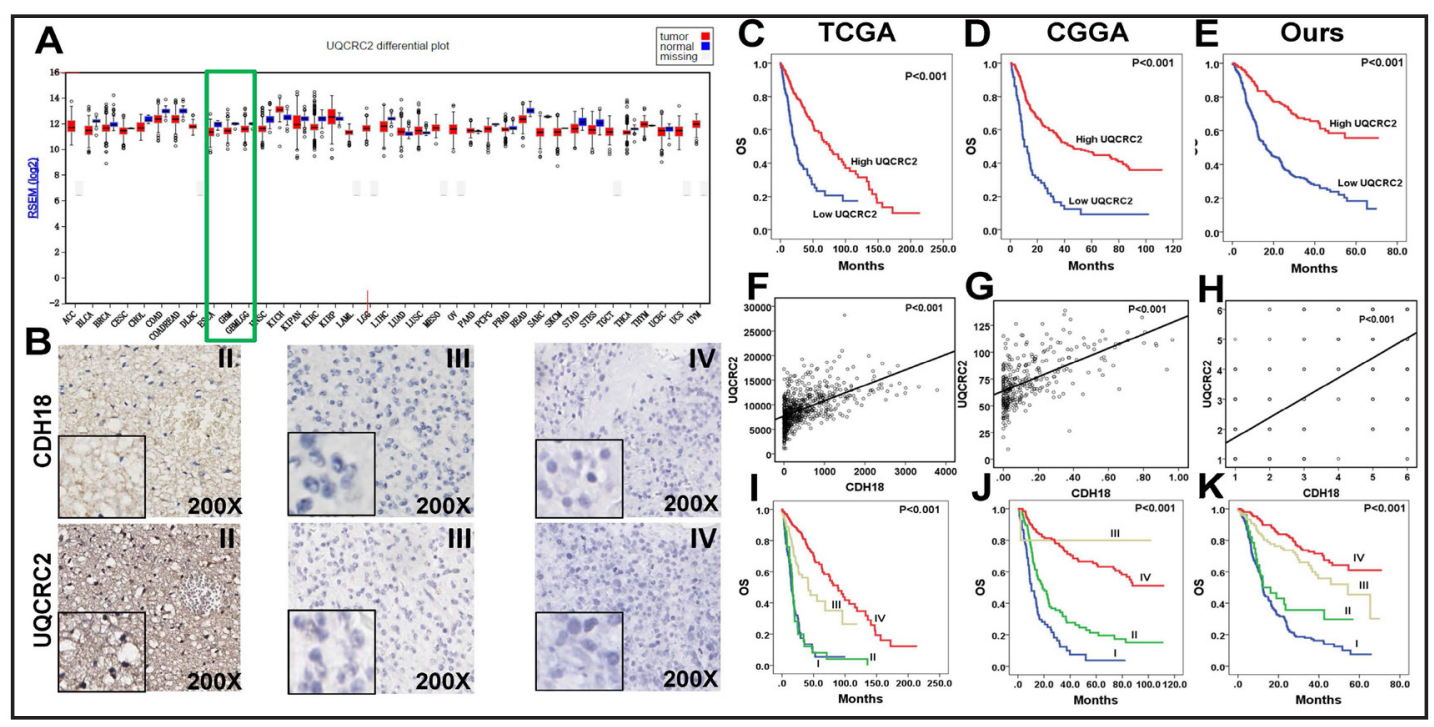

Fig. 6. UQCRC2 is down-regulated in gliomas and positively correlated with CDH18.(A) UQCRC2 expression in FireBrowse database. (B) Immunohistochemical staining of CDH18 and UQCRC2 in gliomas with serial section in Grade II, Grade III and Grade IV gliomas. (C, D, E) Survival analysis in TCGA, CGGA and our glioma cohorts. (F, G, H) Correlation analysis of CDH18 and UQCRC2 in TCGA, CGGA and our glioma cohorts. (I, J, K) Conjoint survival analysis of CDH18 and UQCRC2 in TCGA, CGGA and our glioma cohorts. 


\section{Cellular Physiology Cell Physiol Biochem 2018;48:1755-1770 \\ and Biochemistry Publis. $\begin{aligned} & \text { DOI: 10.1159/000492317 } \\ & \text { (c) } 2018 \text { The Author(s). Published by S. Karger AG, Basel } \\ & \text { www.karger.com/cpb }\end{aligned}$ \\ Bai et al.: Role of $\mathrm{CDH} 18$ in Glioma}

CDH18: 863.3 \pm 18.05 vs 432.7 $\pm 18.21, \mathrm{P}<0.0001$, U251-CDH18+shUQCRC2 vs U251-CDH18: $848.7 \pm 27.14$ vs $421.0 \pm 24.01$, P=0.0003, Fig.5B).

UQCRC2 is down-regulated in gliomas and positively correlated with CDH18

The FireBrowse database showed that the expression of UQCRC2 was significantly higher in LGG than GBM, as well as in LGG compared to normal brain tissue (Fig. 6A). The staining of CDH18 and UQCRC2 with serial sections revealed that expression of CDH18 and UQCRC2 was gradually absent as the WHO grades as gliomas escalated. The staining of UQCRC2 was localized mainly in the cytoplasm and cell membrane (Fig. 6B). Survival analysis of TCGA glioma cohorts and CGGA glioma cohorts showed that patients with high expression of UQCRC2 had a longer OS than those with low expression of UQCRC2 (Fig. 6C, $6 \mathrm{D}, \mathrm{P}<0.001$ ). Also, survival analysis of our glioma cohort showed that patients with high expression of UQCRC2 had a longer OS than those with low expression of UQCRC2 (Fig. 6E, $\mathrm{P}<0.001)$. Correlation analysis of CDH18 and UQCRC2 in TCGA, CGGA and our glioma cohorts showed that CDH18 and UQCRC2 was closely correlated (Fig. $6 \mathrm{H}, \mathrm{P}<0.001$ ). Survival analysis of TCGA, CGGA and our glioma cohorts combined CDH18 and UQCRC2 showed that patients with high expression of CDH18 and UQCRC2 had a longer OS than that with low expression of CDH18 and UQCRC2 (Fig. 6I, 6J, 6K, P<0.001).

\section{Discussion}

For glioma patients, older age at diagnosis was established as a well-known predictor of worse prognosis $[17,24]$. This phenomenon is also observed in experimental brain tumor animal models [25]. In addition, a growing number of studies have demonstrated that several genetic alterations are age-dependent in gliomas, and these genetic alterations play an important role in glioma initiation and progression [18, 19, 26, 27]. Therefore, we first used bioinformatics to analyze TCGA, which is the most influential public database on gliomas, for comparison of genetic alterations between older and younger patients group with gliomas. Combining the results on differential expression of genes, gene ontology, and copy number variations, $C D H 18$ was identified as the target gene significantly downregulated in older group of gliomas. Moreover, public databases revealed that compared to normal brain tissue, $\mathrm{CDH} 18$ was dramatically suppressed in glioma tissues. Interestingly, the expression level of CDH18 is considerably higher in cerebellar hemisphere/cerebellum than other human tissues. Altogether, previous results indicated CDH18 might participate in the carcinogenesis and progression of gliomas.

To our knowledge, this is the first study investigating the role of CDH18 on cancer cell biology and its prognostic value for patients with cancer. An et al., Venkatachalam et al. and Heikinheimo et al. found that $\mathrm{CDH} 18$ gene was mutated in sporadic colorectal cancers and odontogenic keratocytes [14-16]. However, whether $C D H 18$ is an oncogene or tumorsuppressor gene and how $\mathrm{CDH} 18$ participates in carcinogenesis remained unexplored. In this study, we found that the mRNA expression of CDH18 is gradually suppressed from normal brain tissues to GBM in TCGA and CGGA databases, and the findings were corroborated by our cohorts of a large sample size. Survival analyses demonstrated that low expression of CDH18 predicts poorer survival in TCGA and CGGA databases. We performed immunohistochemical staining of CDH18 in our cohort and found the same prognostic value of CDH18 in glioma patients. These observations strongly suggest that CDH18 might negatively regulate the tumorigenesis of gliomas as a tumor suppressor. Hence, we investigated the effects of CDH18 on glioma cell proliferation, invasion, migration and chemo-resistance. Our results demonstrated that $\mathrm{CDH} 18$ overexpression significantly inhibited the invasion and migration ability but not proliferation ability in glioma cell lines. Also, CDH18 overexpression enhanced the cytotoxic effects of TMZ, which is the first-line therapeutic drug for gliomas, on glioma cell lines. We subsequently revealed that $\mathrm{CDH} 18$ suppressed the tumorigenicity of glioma 
cells in vivo. These results demonstrated CDH18 functions as a tumor-suppressor in glioma cell progression and chemo-resistance.

Since there is no previously published research on the mechanism of CDH18 in cancer cell biology for our reference. We employed iTRAQ-based quantitative proteomics to seek the downstream targets of CDH18 in glioma cell lines and identified 143 candidate proteins both changed over 1.5-fold in U87 and U251 glioma cell lines. Subsequent GO analysis demonstrated these differential proteins were mainly enriched in the biological process of intracellular transport, in the cellular content of mitochondria, and in the molecular function of transmembrane transporter. KEGG pathway analysis revealed that the majority of the candidate proteins were correlated with CNS diseases and OXPHOS. Notably, we observed that UQCRC2 existed in all the top five pathways of enrichment. Therefore, UQCRC2 was adopted as the downstream target of CDH18 in the current study. Further in vitro assays demonstrated that down-regulation of UQCRC2 partly reversed the inhibition of invasion/ migration ability and chemo-resistance in CDH18 overexpressed glioma cell lines. Moreover, public database showed that UQCRC2 is considerably suppressed in glioma tissues compared to normal brain tissues. We further revealed that UQCRC2 was positively correlated with CDH18 on the mRNA level and protein level in glioma cohorts with large sample size. Survival analysis demonstrated that combined CDH18/UQCRC2 biomarkers significantly influenced the prognosis of glioma patients. These findings suggested UQCRC2 might function as a key protein downstream of CDH18. UQCRC2 is one of the two core proteins of the ubiquinol-cytochrome c reductase complex (complex III), which constitutes a part of the mitochondrial respiratory chain $[28,29]$. It was recently reported that homozygous missense mutation in UQCRC2 might cause mitochondrial complex III deficiency, which is a relatively rare disease [28]. Putignani et al. observed that the content of UQCRC2 in breast cancer cell was significantly decreased compared to that in normal epithelial cells [30, 31], which is in accordance with our findings on gliomas. In recent years, a growing number of studies provided new insight into the role of mitochondria in cancer and demonstrated that impaired bioenergetic function of mitochondria is a hallmark of tumorigenesis [32, 33]. It has been reported that mitochondrial alteration is correlated with cancer cell motility and invasiveness, as well as chemo-resistance [34-36]. We inferred that CDH18 might influence the function of mitochondria via UQCRC2, and thus inhibited the invasiveness and chemoresistance of glioma cells. Our data shed light on a new perspective concerning the role of CDH18 in gliomas, and further investigations are needed to gain more insight on the relationship between $\mathrm{CDH} 18$, mitochondria and glioma carcinogenesis.

Last but not least, our results also suggested several new aspects of potential significance to be further investigated. First, molecular subgroups based on IDH mutations, TERT promoter mutations, and $1 \mathrm{p} / 19 \mathrm{q}$ co-deletion of gliomas were charaterized by distinct mechanisms of pathogenesis $[2,37]$. Since CDH18 is firstly studied in gliomas, whether its expression and functions are altered between different molecular subgroups of gliomas remains unexplored. Second, it was revealed that the levels of OXPHOS are down-regulated in glioma cells due to several reasons, one of which is the direct loss of function of components of OXPHOS, such as complex II, III and IV [38, 39]. Since UQCRC2 is a core component of complex III, we infer that the down-regulation of CDH18 in gliomas inhibits the expression of UQCRC2 protein, which contributes to the loss of function of complex III and decreased activity of OXPHOS in gliomas. Further investigations are needed to clarify the metabolic change caused by CDH18 and UQCRC2 in gliomas. Third, the ketogenic diet is a high-fat and low-carbohydrate diet that used in treating gliomas for years [40]. Our results explain to some extent why a ketogenic diet could be an optional therapy for gliomas because unlike normal brain cells, the tumor cells have relatively low expression of CDH18 and UQCRC2, which contributes to the lack of a functional OXPHOS system necessary for metabolism of ketones for energy production [37, 39]. However, this hypothesis for ketogenic diet in gliomas needs further investigation.

In summary, our study identified a novel tumor-suppressor, CDH18, in gliomas. We investigated the influence of CDH18 on cancer cell biology and its prognostic value for the first time. We also demonstrated that CDH18 exerted its tumor-suppressor role via UQCRC2 
in glioma cells. Our findings suggested CDH18 might serve as a therapeutic target for treating gliomas.

\section{Acknowledgements}

This work wassupported by the National Natural Science Foundation of China(81702465), the Youth Innovation Fund of The First Affiliated Hospital of Zhengzhou University to Zhenyu Zhang, the Youth Innovation Fund of The First Affiliated Hospital of Zhengzhou University to Ya-hui Bai, the Youth Innovation Fund of The First Affiliated Hospital of Zhengzhou University to Yan-min Wang, the Science and Technology Program of Henan Province to Jinqiao Zhou, and the Medical Science and Technology Program of Henan Province to Jin-qiao Zhou (201602058). We would like to thank Jiesheng Lu (Laboratory of Personalized Genomic Medicine, Columbia University in the City of New York, USA) for assistance with reviewing this article. Ya-hui Bai, Yun-bo Zhan, Bin Yu: Conception and helped design of research, satistical analysis of data, drafting and modifying of manuscript, final approval of the version to be published. Yun-bo Zhan assembled all the final figures (Figure1-6 and Supplementary Fig. 1-3). Wei-Wei Wang, Li Wang: Pathological diagnosis and immunohistochemistry. Wei-Wei Wang generated figure of immunohistochemistry. Jin-qiao Zhou, Ruo-kun Chen, Feng-jiang Zhang: Perform cell culture and transfection, CCK-8 assay, transwell migration assay, wound healing assay, clonogenic assay under temozolomide(TMZ) presence and tumorigenicity assay in nude mice, generate above-mentioned data and corresponding figure. Yan-min Wang, Jun Liu, Jian-ji Bao: Perform RNA extraction, reverse transcription, and quantitative real-time PCR (qRT PCR), western Blotting, iTRAQ sample preparation, two dimensional LCMS/MS and proteomics data analysis, generated above-mentioned data and corresponding figure. Xin-wei Zhao, Wen-chao Duan: Follow-up visits and acquisition of tissue specimens and clinical data. Zhen-Yu Zhang, Xian-zhi Liu: Conception and design of research, editing manuscript, final approval of the version to be published.

\section{Disclosure Statement}

Conflict of interests relevant to this article was not reported.

\section{References}

1 Cui R, Guan Y, Sun C, Chen Y, Bao Y, Li G: A tumor-suppressive microRNA, miR-504, inhibits cell proliferation and promotes apoptosis by targeting FOXP1 in human glioma. Cancer Lett 2016;374:1-11.

$>2$ Louis DN, Perry A, Reifenberger G, Deimling AV, Figarella-Branger D, Cavenee, WK: The 2016 World Health Organization Classification of Tumors of the Central Nervous System: a summary. Acta Neuropathol 2016;131:803-820.

-3 Tseliou M, Al-Qahtani A, Alarifi S, Alkahtani SH, Stournaras C, Sourvinos G: The role of RhoA, RhoB and RhoC GTPases in cell morphology, proliferation and migration in human cytomegalovirus (HCMV) infected glioblastoma cells. Cell Physiol Biochem 2016;38:94-109.

-4 Zhang BL, Dong FL, Guo TW, Gu XH, Huang LY, Gao DS: MiRNAs mediate GDNF-induced proliferation and migration of glioma cells. Cell Physiol Biochem 2017;44:1923-1938.

5 Nollet F, Kools P, van Roy F: Phylogenetic analysis of the cadherin superfamily allows identification of six major subfamilies besides several solitary members. J Mol Biol 2000;299:551-572.

6 Takeichi M: Cadherin cell adhesion receptors as a morphogenetic regulator. Science 1991;251:1451-1455.

7 Thiery JP: Epithelial-mesenchymal transitions in tumour progression. Nat Rev Cancer 2002;2:442-454.

8 Redies C, Hertel N, Hübner CA: Cadherins and neuropsychiatric disorders. Brain Res 2012;1470:130-144.

9 Chen X, Long F, Cai B, Chen X, Chen G: A novel relationship for schizophrenia, bipolar and major depressive 


\section{Cellular Physiology Cell Physiol Biochem 2018;48:1755-1770

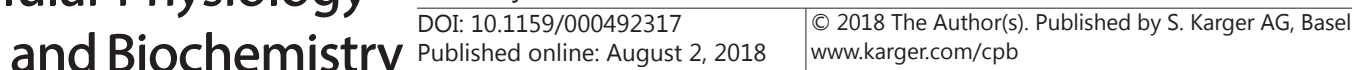

disorder Part 5: a hint from chromosome 5 high density association screen. Am J Transl Res 2017;9:24732491.

10 Kong SW, Collins CD, Motohashi YS, Holm LA, Campbell MG, Lee IH: Characteristics and predictive value of blood transcriptome signature in males with autism spectrum disorders. PLoS One 2012;7:e49475.

11 Redies C, Hertel N, Hübner CA: Cadherins and neuropsychiatric disorders. Brain Res 2012;1470:130-144.

12 Terracciano A, Tanaka T, Sutin AR, Sanna S, Deiana B, Lai S: Genome-wide association scan of trait depression. Biol Psychiatry 2010;68:811-817.

$\checkmark 13$ Anitha A, Thanseem I, Nakamura K, Yamada K, Iwayama Y, Toyota T: Protocadherin $\alpha$ (PCDHA) as a novel susceptibility gene for autism. J Psychiatry Neurosci 2013;38:192-198.

-14 An CH, Je EM, Yoo NJ, Lee SH: Frameshift mutations of cadherin genes DCHS2, CDH10 and CDH24 genes in gastric and colorectal cancers with high microsatellite instability. Pathol Oncol Res 2015;21:181-185.

15 Venkatachalam R, Verwiel ET, Kamping EJ, Hoenselaar E, Görgens H, Schackert HK: Identification of candidate predisposing copy number variants in familial and early-onset colorectal cancer patients. Int J Cancer 2011;129:1635-1642.

16 Heikinheimo K, Jee KJ, Morgan PR, Nagy B, Knuutila S, Leivo I: Genetic changes in sporadic keratocystic odontogenic tumors (odontogenic keratocysts). J Dent Res 2007;86:544-549.

17 Burger PC, Vogel FS, Green SB, Strike TA: Glioblastoma multiforme and anaplastic astrocytoma. Pathologic criteria and prognostic implications. Cancer 1985;56:1106-1111.

18 Hartmann C, Hentschel B, Wick W, Capper D, Felsberg J, Simon M: Patients with IDH1 wild type anaplastic astrocytomas exhibit worse prognosis than IDH1-mutated glioblastomas, and IDH1 mutation status accounts for the unfavorable prognostic effect of higher age: implications for classification of gliomas. Acta Neuropathol 2010;120:707-718.

19 Walsh KM, Rice T, Decker PA, Kosel ML, Kollmeyer T, Hansen HM: Genetic variants in telomerase-related genes are associated with an older age at diagnosis in glioma patients: evidence for distinct pathways of gliomagenesis. Neuro Oncol 2013;15:1041-1047.

20 Dai DL, Martinka M, Li G: Prognostic significance of activated Akt expression in melanoma: a clinicopathologic study of 292 cases. J Clin Oncol 2005;23:1473-1482.

21 Shen J, Wang B, Zhang T, Zhu N, Wang Z, Jin J, He Y, Hu M: Suppression of Non-Small Cell Lung Cancer Growth and Metastasis by a Novel Small Molecular Activator of RECK. Cell Physiol Biochem 2018;45:18071817.

22 Anders S, Huber W: Differential expression analysis for sequence count data. Genome Biol 2010;11:R106.

23 Beroukhim R: Assessing the significance of chromosomal aberrations in cancer: methodology and application to glioma. Proc Natl Acad Sci USA 2007;104:20007-20012.

24 Siker ML, Wang M, Porter K, Nelson DF, Curran WJ, Michalski JM: Age as an independent prognostic factor in patients with glioblastoma: A Radiation Therapy Oncology Group and American College of Surgeons National Cancer Data Base comparison. J Neurooncol 2011;104:351-356.

25 Ladomersky E, Zhai L, Gritsina G, Genet M, Lauing KL, Wu M: Advanced age negatively impacts survival in an experimental brain tumor model. Neurosci Lett 2016;630:203-208.

-26 Batchelor TT, Betensky RA, Esposito JM, Pham LD, Dorfman MV, Piscatelli N: Age-dependent prognostic effects of genetic alterations in glioblastoma. Clin Cancer Res 2004;10:228-233.

-27 Hartmann C, Meyer J, Balss J, Capper D, Mueller W, Christians A: Type and frequency of IDH1 and IDH2 mutations are related to astrocytic and oligodendroglial differentiation and age: a study of 1, 010 diffuse gliomas. Acta Neuropathol 2009;118:469-474.

28 Miyake N, Yano S, Sakai C, Hatakeyama H, Matsushima Y, Shiina M: Mitochondrial complex III deficiency caused by a homozygous UQCRC2 mutation presenting with neonatal-onset recurrent metabolic decompensation. Hum Mutat 2013;34:446-452.

29 Gaignard P, Eyer D, Lebigot E, Oliveira C, Therond P, Boutron A: UQCRC2 mutation in a patient with mitochondrial complex III deficiency causing recurrent liver failure, lactic acidosis and hypoglycemia. J Hum Genet 2017;62:729-731.

-30 Putignani L, Raffa S, Pescosolido R, Aimati L, Signore F, Torrisi MR: Alteration of expression levels of the oxidative phosphorylation system (OXPHOS) in breast cancer cell mitochondria. Breast Cancer Res Treat 2008;110:439-452.

-31 Putignani L, Raffa S, Pescosolido R, Rizza T, Del CF, Leone L: Preliminary evidences on mitochondrial injury 


\section{Cellular Physiology Cell Physiol Biochem 2018:48:1755-1770

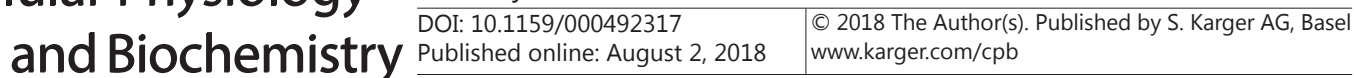

Bai et al.: Role of $\mathrm{CDH} 18$ in Glioma

and impaired oxidative metabolism in breast cancer. Mitochondrion 2012;12:363-369.

-32 Brandon M, Baldi P, Wallace DC: Mitochondrial mutations in cancer. Oncogene 2006;25:4647-4662.

-33 Isidoro A, Martínez M, Fernández PL, Ortega AD, Santamaría G, Chamorro M: Alteration of the bioenergetic phenotype of mitochondria is a hallmark of breast, gastric, lung and oesophageal cancer. Biochem J 2004;378:17-20.

34 Che TF, Lin CW, Wu YY, Chen YJ, Han CL, Chang YL: Mitochondrial translocation of EGFR regulates mitochondria dynamics and promotes metastasis in NSCLC. Oncotarget 2015;6:37349-37366.

-35 Zhao J, Zhang J, Yu M, Xie Y, Huang Y, Wolff DW: Mitochondrial dynamics regulates migration and invasion of breast cancer cells. Oncogene 2013;32:4814-4824.

36 Zheng HC: The molecular mechanisms of chemoresistance in cancers. Oncotarget 2017;8:59950-59964.

-37 Eckel-Passow JE, Lachance DH, Molinaro AM, Walsh KM, Decker PA, Sicotte H, Pekmezci M, Rice T, Kosel ML, Smirnov IV, Sarkar G, Caron AA, Kollmeyer TM, Praska CE, Chada AR, Halder C, Hansen HM, McCoy LS, Bracci PM, Marshall R, Zheng S, Reis GF, Pico AR, O’Neill BP, Buckner JC, Giannini C, Huse JT, Perry A, Tihan T, Berger MS, Chang SM, Prados MD, Wiemels J, Wiencke JK, Wrensch MR, Jenkins RB: Glioma Groups Based on 1p/19q, IDH, and TERT Promoter Mutations in Tumors. N Engl J Med 2015;372:2499-2508.

-38 Feichtinger RG, Weis S, Mayr JA, Zimmermann F, Geilberger R, Sperl W, Kofler B: Alterations of oxidative phosphorylation complexes in astrocytomas. Glia 2014;62:514-525.

39 Strickland M, Stoll EA: Metabolic Reprogramming in Glioma. Front Cell Dev Biol 2017;26:5-43.

40 Seyfried TN, Flores R, Poff AM, D’Agostino DP, Mukherjee P: Metabolic therapy: a new paradigm for managing malignant brain cancer. Metabolic therapy: a new paradigm for managing malignant brain cancer. Cancer Lett 2015;356:289-300. 